\title{
A polymorphism within the mismatch repair gene predicts prognosis and adjuvant chemotherapy benefit in gastric cancer
}

\author{
Xiaohui Zhao ${ }^{1,2} \cdot$ Dongfang Dai $^{1} \cdot$ Xiaoqin $\mathrm{Li}^{1} \cdot$ Bo Shen $^{3} \cdot$ Xiaofeng Chen ${ }^{4} \cdot$ Yongqian Shu ${ }^{4} \cdot$ Deqiang Wang ${ }^{1,4}$ (I)
}

Received: 20 February 2019 / Accepted: 3 April 2019 / Published online: 15 April 2019

(c) The International Gastric Cancer Association and The Japanese Gastric Cancer Association 2019

\begin{abstract}
Background Defective mismatch repair (dMMR) and microsatellite instability (MSI) correlate with gastric cancer (GC) outcome. We hypothesized that MMR genetic polymorphisms that have low-penetrant effects but may cause heterogeneous MMR capability among individuals also affect GC outcome.

Methods The polymorphisms rs1800734 in MLH1, rs2303428 and rs3732183 in MSH2, rs735943 in EXO1, and rs11797 in TREX1 were selected and analyzed in independent discovery and validation sets that included 167 and 593 patients, respectively. MSI was determined.

Results In both the discovery and validation sets, the rs $2303428 T C+C C$ genotype correlated with poor overall survival $(\mathrm{OS})$ in non-cardia $(P<0.05)$ but not in cardia GC. Multivariate models showed that for OS of patients with non-cardia GC, the rs2303428 $T C+C C$ genotype was an independent predictor in the validation set (HR $1.54 ; 95 \%$ CI 1.02-2.32; $P=0.040$ ) and had a trend to be an independent predictor in the discovery set (HR 1.70; 95\% CI 0.96-3.01; $P=0.067)$. Furthermore, in both patient sets, fluoropyrimidines-based adjuvant chemotherapy improved OS for non-cardia patients with the rs 2303428 $T C+C C$ genotype (HR 0.14; 95\% CI 0.04-0.57; $P=0.006$; and HR 0.29; 95\% CI 0.15-0.58; $P<0.001$, respectively) but not for those with the TT genotype. The rs 2303428 genotypes were not associated with MSI frequency. The rs2303428 $T C+C C$ genotype correlated with reduced expressions for thymidylate synthetase, P-glycoprotein and ERCC1 $(P<0.05)$ in non-cardia GC.
\end{abstract}

Conclusions The rs2303428 genotypes may predict prognosis and adjuvant chemotherapy benefit in non-cardia GC patients.

Keywords Polymorphism · Mismatch repair · Microsatellite instability · Gastric cancer · Adjuvant chemotherapy

Electronic supplementary material The online version of this article (https://doi.org/10.1007/s10120-019-00962-8) contains supplementary material, which is available to authorized users.

Yongqian Shu

shuyongqian@csco.org.cn

$\triangle$ Deqiang Wang

deqiang_wang@aliyun.com

1 Department of Medical Oncology, The Cancer Therapy Center, Affiliated Hospital of Jiangsu University, Road Jiefang, Zhenjiang 212001, Jiangsu, China

2 Department of Pathology, Affiliated Hospital of Jiangsu University, Zhenjiang, Jiangsu, China

3 Department of Medical Oncology, The Affiliated Cancer Hospital of Nanjing Medical University, Nanjing, China

4 Department of Medical Oncology, The First Affiliated Hospital of Nanjing Medical University, Road Guangzhou, Nanjing 210029, China

\section{Introduction}

Gastric cancer (GC) is the fifth most common cancer and the third-leading cause of cancer-related death worldwide, with a particularly high prevalence in China [1,2]. GC is a complex disease with significant genetic heterogeneity among patients [3], which limits the use of personalized therapy and contributes to its high mortality. Identifying novel prognostic and predictive markers is crucial to improve patient outcomes of GC.

DNA mismatch repair (MMR) is critical for genome stability and defective MMR (dMMR) can result in microsatellite instability (MSI) phenotype [4]. MSI or dMMR is a prognostic predictor in patients with stage II colon cancer and may also decrease efficacy of fluoropyrimidines (FU)based adjuvant chemotherapy in these patients [5, 6]. In GC, retrospective studies also find that FU-based adjuvant chemotherapy improves survival in microsatellite stable (MSS) 
patients but not in MSI patients [7, 8]. Despite this, one study described no association of MSI with chemotherapy benefit [9]. More conflicting results are reported about the prognostic role of MSI or dMMR [7-10].

Except the debate about the association of MSI or dMMR with outcome, there are some other limitations for the use of MSI and dMMR as biomarkers. First, the present accepted methods for testing MSI (PCR) and dMMR (immunohistochemistry, IHC) still need to be standardized and validated. Especially, the results from IHC or PCR are sometimes difficult to interpret, interobserver variability may occur and therefore MSI or dMMR may be misdiagnosed [11, 12]. Second, intratumoural heterogeneity can cause different detection results and such heterogeneity in MSI GC was reported to correlate with non-responsiveness of immune therapy [13]. Third, varied incidences of MSI or dMMR were reported in GC, which may be partly dependent on testing methods and sample types [14]. Furthermore, some genetic defects may impact MMR function or activity other than MMR protein expression level [15]. Specifically, common polymorphisms of MMR genes with a low-penetrant effect that may be also insufficient to induce MSI can cause heterogeneous MMR capability among individuals, which correlates with cancer risk and clinical outcome [16-29].

We previously studied the association of polymorphisms in MMR genes with GC risk [17, 18]. These polymorphisms were required to have a minor allele frequency of greater than $20 \%$ among Chinese and needed to be associated with cancer risk or clinical outcome in previous reports with a potential function. Finally, five polymorphisms in four genes of the MMR system were selected, including rs1800734 in $M L H 1$, rs2303428 and rs3732183 in MSH2, rs735943 in EXO1, and rs11797 in TREX1.

The rs1800734 A-allele has a lower binding affinity for transcription factors and an increased risk of hypermethylation for the $M L H 1$ promoter than the $G$-allele, and therefore is associated with morbidity of many cancers $[19,20]$. The rs2303428 and rs3732183 polymorphisms may impact the alternative splicing of $M S H 2$ mRNA to increase cancer risk [16-18, 21-24]. The rs3732183 $G$-allele was also an independent prognostic factor in colorectal cancer [25]. The rs11797 and rs735943 polymorphisms are independent predictor for certain outcomes in pancreatic cancer patients who received preoperative chemoradiotherapy; their functions suggest correlating with transcriptional regulation and protein function [26].

We demonstrate an association of some of these polymorphisms with GC risk [17, 18]. Given the existing functional and clinical evidences, we hypothesized in this study that these polymorphisms may also affect GC outcome, which had yet to be reported. The rationality of this hypothesis is strengthened by the advantages of polymorphisms as tumor biomarkers, including common frequency, high stability and reliability, ease of analysis, short test time, low genotyping costs, and testing independent of tissue.

\section{Methods}

\section{Patients}

The discovery set included 167 GC patients diagnosed in 2012 at the First Affiliated Hospital of Nanjing Medical University. The validation set enrolled $593 \mathrm{GC}$ patients screened in a prospectively designed database who were diagnosed at the Affiliated Hospital of Jiangsu University between 2012 and 2015. Eligibility criteria for all patients included ages from 18 to 75 years, available untreated peripheral blood sample, and pathological diagnosis of gastric adenocarcinoma. Exclusion criteria included insufficient clinicopathological data and prior history of anti-tumor therapy (including neoadjuvant therapy) such as chemotherapy, radiotherapy and biological target therapy. The American Joint Committee on Cancer (AJCC) criteria was used for clinical and clinicopathologic classification and stage. Patient follow-up was completed on December 31, 2017. Informed consent was obtained from all patients and the research protocol was approved by the hospital ethics committee.

\section{Candidate polymorphisms}

We searched public databases of NCBI-Pubmed and dbSNP (http://www.ncbi.nlm.nih.gov) to identify potentially functional polymorphisms within genes involved in MMR. The select criteria and enrolled polymorphisms had been described in introduction.

\section{DNA extraction and genotyping}

DNA was extracted from peripheral blood lymphocytes and genotyping was conducted by Taqman allelic discrimination assays as previously described [18].

\section{MSI assessment}

Genomic DNA was extracted from 196 macrodissected cancer and noncancer tissues. MSI status was determined by single fluorescent multiplex PCR using five quasimonomorphic mononucleotide repeat markers (BAT-25, BAT-26, NR-21, NR-24 and NR-27) [30]. Tumors were either classified as MSI when more than one marker was unstable, or MSS. 


\section{Immunohistochemistry analysis}

Antibodies (Abcam, UK) for thymidylate synthetase (TS), P-glycoprotein (Pgp), ERCC1 and HER2 were used for immunohistochemistry (IHC) with a 2-step protocol and immunohistochemical score was assessed as previously reported [31, 32].

\section{Statistical analysis}

The primary endpoint was overall survival (OS) which was defined as the interval between pathological diagnosis or surgery and cancer-related death or the last date of follow-up. The second endpoint was recurrence-free survival (RFS) which was defined as the duration from the date of operation to the date of recurrence, metastasis, or follow-up termination. The Hardy-Weinberg equilibrium for each polymorphism was tested by the goodness-of-fit $\chi^{2}$ test. Comparisons for baseline characteristics used the $\chi^{2}$ test. The Kaplan-Meier method and the Log-rank test were used for survival analysis. Univariate and multivariate Cox proportional hazard regression models were used to calculate hazard ratio (HR) and 95\% confidence intervals (CIs) of variables. Two-sided $P<0.05$ was considered as statistically significant. Analyses were conducted using SPSS 19.0 (SPSS, Chicago, IL, USA).

\section{Results}

\section{Patient characteristics and genotype frequencies}

The proportion of patients with each characteristic (except sex and histology grade) was significantly different between the discovery and validation sets, verifying that these two patient cohorts were independent (Table 1). Genotype frequencies of the discovery and validation sets are presented in Table 2. All genotype distributions among patients in both discovery and validation sets were in Hardy-Weinberg equilibrium.

\section{Associations of genotypes with prognosis}

In the discovery set, the rs2303428 TC+CC and rs11797 $G A+A A$ genotypes were significantly associated with poor OS $(P<0.05 ;$ Fig. 1$)$. In the independent validation set, we further confirmed the prognostic association for the rs2303428 TC+CC genotype, but not for the rs11797 $G A+A A$ genotype (Fig. 2a and Table 3). Interestingly, the prognostic role of the rs2303428 TC $+C C$ genotype in our subgroup analyses according to patient characteristics was observed in non-cardia $(P<0.05)$ but not in cardia GC
Table 1 Patient characteristics

\begin{tabular}{lccc}
\hline Characteristic & \multicolumn{2}{l}{ No. of patients $(\%)$} & P value \\
\cline { 2 - 3 } & Discovery set & Validation set & \\
\hline Age (years) & $53(31.7)$ & $263(44.4)$ & 0.004 \\
$\geq 65$ & $114(68.3)$ & $330(55.6)$ & \\
$<65$ & & & \\
Sex & $122(73.1)$ & $427(72.0)$ & 0.790 \\
Male & $45(26.9)$ & $166(28.0)$ & \\
Female & & & \\
Tumor location & $60(35.9)$ & $288(48.6)$ & 0.004 \\
Cardia & $107(64.1)$ & $305(51.4)$ & \\
Non-cardia & & & \\
Histology grade & $95(56.9)$ & $297(50.1)$ & 0.120 \\
III & $72(43.1)$ & $296(49.9)$ & \\
I/II & & & \\
Histology type & $5(3.0)$ & $64(10.8)$ & 0.002 \\
MAC/SRCC & $162(97.0)$ & $529(89.2)$ & \\
Other ADC & & & \\
TNM stage & $117(70.1)$ & $366(61.7)$ & 0.048 \\
III/IV & $50(29.9)$ & $227(38.3)$ & \\
I/II & & & \\
\hline
\end{tabular}

$M A C$ mucinous adenocarcinoma, SRCC signet ring cell carcinoma, $A D C$ adenocarcinoma

$(P>0.05)$ in both the discovery and validation sets (Supporting Fig. 1A, B, and Fig. 2b, c). The multivariate models showed that for OS of patients with non-cardia GC, the rs $2303428 T C+C C$ genotype was an independent predictor in the validation set (HR 1.54; 95\% CI 1.02-2.32; $P=0.040)$ and tended to be an independent predictor in the discovery set (HR 1.70; 95\% CI 0.96-3.01; $P=0.067$; Supporting Table 1).

Moreover, we combined the discovery and validation sets to analyze the prognostic role of the MMR genotypes in all patients. We confirmed the poor OS (Supporting Fig. 2A, B) and the independent predictive role (Supporting Table 1) of the rs2303428 TC $+C C$ genotype in noncardia GC. We still found no prognostic association for other polymorphisms (Supporting Fig. 3).

RFS was available in 119 patients of the discovery set and in 391 patients of the validation set. The rs 2303428 $T C+C C$ genotype correlated or tended to correlate with poor RFS in non-cardia but not in cardia GC in both the discovery and validation sets and in all patients (Supporting Fig. 4). The multivariate models in all patients with non-cardia GC found that the rs2303428 TC $+C C$ genotype tended to be an independent predictor for RFS (HR 1.48; 95\% CI 0.97-2.25; $P=0.069$; Supporting Table 2). No significant correlation was found between RFS and other polymorphisms (data not shown). 
Table 2 Polymorphisms examined and genotype frequency

\begin{tabular}{lllccl}
\hline Gene & SNP & Patient set & \multicolumn{2}{l}{ No. of patients ${ }^{\mathrm{a}}(\%)$} & \\
\cline { 3 - 6 } & & & Major/major allele & Major/minor allele & Minor/minor allele \\
\hline \multirow{2}{*}{ MLH1 } & \multirow{2}{*}{ rs1800734 } & Discovery & $52(31.1)$ & $90(53.9)$ & $25(15.0)$ \\
& & Validation & $203(35.6)$ & $288(50.5)$ & $79(13.9)$ \\
MSH2 & \multirow{2}{*}{ rs2303428 } & Discovery & $101(60.5)$ & $56(33.5)$ & $10(6.0)$ \\
& & Validation & $234(40.6)$ & $275(47.7)$ & $67(11.7)$ \\
& \multirow{2}{*}{ rs3732183 } & Discovery & $56(33.5)$ & $88(52.7)$ & $23(13.8)$ \\
& & Validation & $220(38.2)$ & $271(47.0)$ & $85(14.8)$ \\
EXO1 & \multirow{2}{*}{ rs735943 } & Discovery & $86(51.5)$ & $70(41.9)$ & $11(6.6)$ \\
& & Validation & $307(53.3)$ & $233(40.5)$ & $36(6.2)$ \\
& \multirow{2}{*}{ rs11797 } & Discovery & $86(51.5)$ & $64(38.3)$ & $17(10.2)$ \\
& & Validation & $291(50.5)$ & $236(41.0)$ & $49(8.5)$
\end{tabular}

${ }^{a}$ Less than $4 \%$ of the patients failed in genotyping in the validation set

$S N P$ single nucleotide polymorphism, $r$ s reference SNP identification number

\section{Associations of the rs2303428 genotypes with the benefit of adjuvant chemotherapy}

We further analyzed the predictive role of the rs 2303428 genotypes. Patients were required to have diseases of stage IB-III after D2 radical gastrectomy and receive more than one course FU [5-fluorouracil, capecitabine or S-1 (Tegafur, Gimeracil and Oteracil Potassium Capsules)]based adjuvant therapy or surgery alone. Characteristics of these patients are shown in Supporting Table 3.

In both the discovery (Supporting Fig. 1C-F) and validation (Fig. 3) sets, FU-based adjuvant chemotherapy significantly improved OS for the rs2303428 TC +CC genotype in non-cardia $(P<0.05)$, but not in cardia patients $(P>0.05)$. No significant results were found for the rs2303428 TT genotype. The multivariate models showed that FU-based chemotherapy was an independent predictor for OS in patients with non-cardia GC and the rs $2303428 T C+C C$ genotype in both the discovery and validation sets (HR $0.14 ; 95 \%$ CI $0.04-0.57 ; P=0.006$; and HR 0.29 ; 95\% CI $0.15-0.58 ; P<0.001$, respectively; Supporting Table 4).

The predictive role of the rs 2303428 genotypes were also analyzed in all patients. We confirmed the OS benefit (Supporting Fig. 2C-F) from adjuvant chemotherapy and the independent predictive role (Supporting Table 4) of adjuvant chemotherapy in patients with non-cardia GC and the rs2303428 TC $+C C$ genotype.

Furthermore, adjuvant chemotherapy improved RFS for patients with non-cardia GC and the rs2303428 TC+CC genotype in both the discovery and validation sets and in all patients (Supporting Fig. 5). The multivariate models in all patients with non-cardia GC and the rs $2303428 T C+C C$ genotype found that adjuvant chemotherapy was an independent predictor for RFS (HR 0.36; 95\% CI 0.21-0.63; $P<0.001$; Supporting Table 5).

\section{Associations of the rs2303428 genotypes with MSI}

As shown in Supporting Table 6, the frequencies of MSI were $10.7 \%$ and $11.6 \%$ for the rs $2303428 T T$ and $T C+C C$ genotypes, respectively $(P=0.845)$. Stratified by tumor locations, there were also no significant results.

\section{Associations of the rs 2303428 genotypes with biomarkers of drug-resistance}

IHC was conducted in 361 tumor samples. Typical protein expressions of TS, Pgp, ERCC1 and HER2 are shown in Supporting Fig. 6. As shown in Table 4, patients with the rs2303428 TC $+C C$ genotype had significantly lower positive rates for TS, Pgp and ERCC1 than those with the TT genotype in non-cardia $(P=0.038,0.017$ and 0.015 , respectively) but not in cardia tumors $(P>0.05)$.

\section{Discussion}

Surgery and subsequent adjuvant chemotherapy have been established as an effective treatment for patients with earlystage GC [33, 34]. Unfortunately, after optimal multimodality therapy, up to $30-40 \%$ of patients will relapse within 5 years $[33,34]$. There are no validated prognostic or predictive biomarkers for GC patients who receive adjuvant chemotherapy, and current patient selection is based mainly on postoperative pathological staging. To our knowledge, our study is the first to report the prognostic and predictive roles of the MMR genotypes in GC. Although prospective validation is necessary, our findings have the potential to improve patient selection for adjuvant chemotherapy and spare a large number of GC patients from unnecessary therapy.

We found that the rs2303428 TC+CC genotype correlated with poor OS and RFS, but predicted the 
Fig. 1 Overall survival in patients from the discovery set by MMR genotypes. MMR DNA mismatch repair
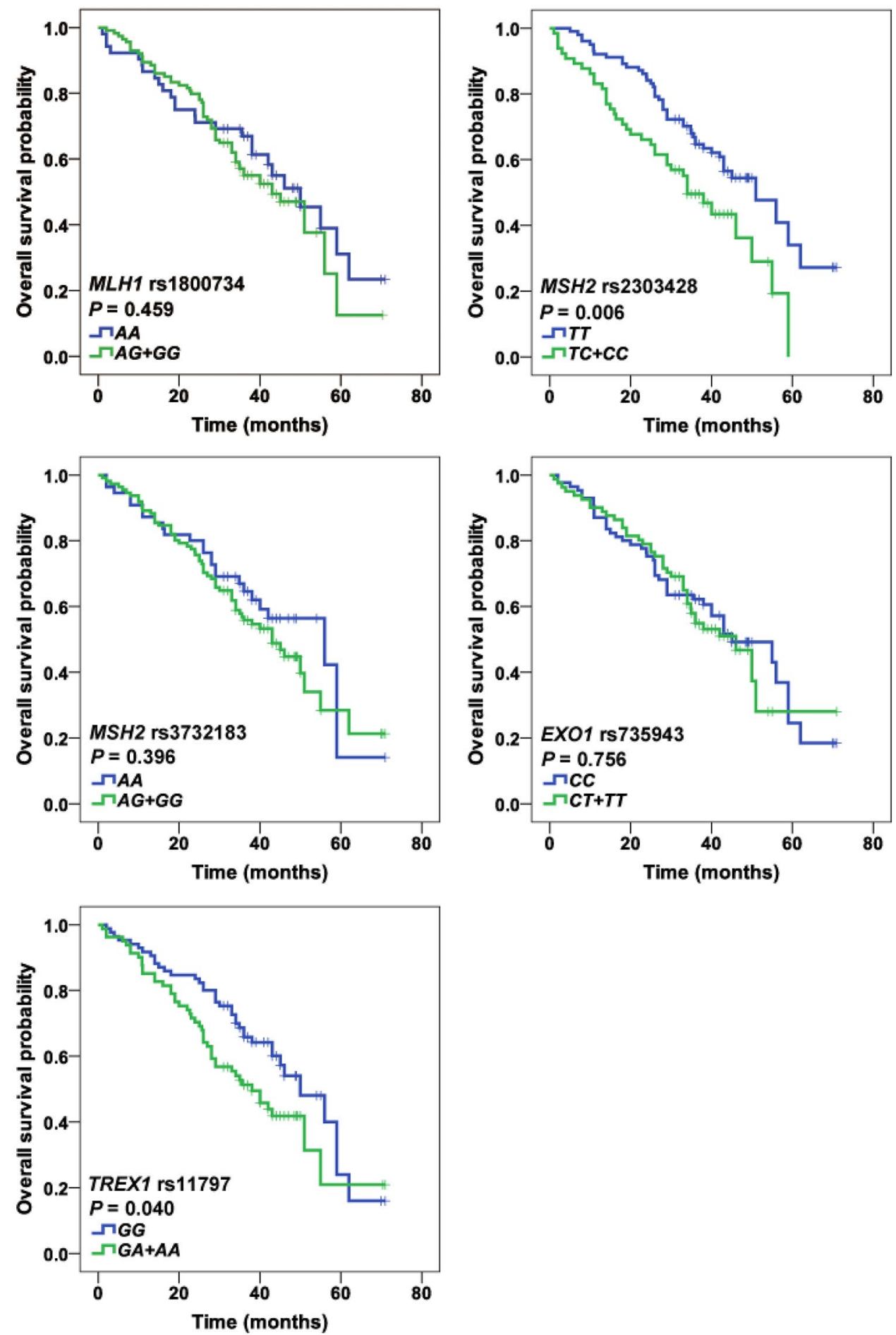

benefit of adjuvant chemotherapy in non-cardia patients. The rs2303428 $\mathrm{C}$-allele may induce partial skipping of the $\mathrm{MSH} 2$ exon 13, which may result in a defective MSH2 protein and subsequent MSI [16, 21]. Nevertheless, our study failed to find a correlation between the rs2303428 $C$-allele and the MSI phenotype, which is consistent with our previous results in 314 GC patients [18], suggesting its low-penetrant role or other mechanisms. Recently, Liu et al. [35] found that the $M L H 1$ rs 1800734 polymorphism in colorectal cancer could serve as a distal enhancer for the DCLK3 gene by a long-range chromatin interaction, which may promote an epithelial-to-mesenchymal transition. Notably, their findings were based on the MSS tumors. In this study, we also found a significantly negative association between the rs 2303428 

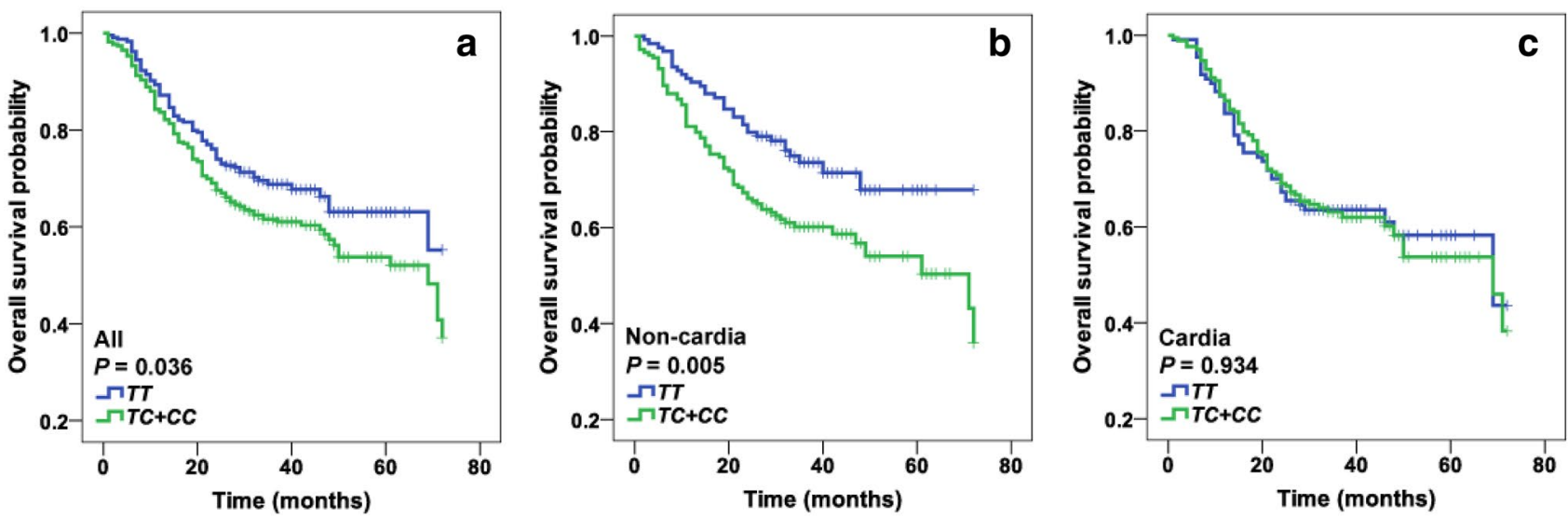

Fig. 2 Overall survival in patients from the validation set by the rs 2303428 genotypes and tumor locations

Table 3 Overall survival by the rs 2303428 and rs11797 genotypes in the validation set

\begin{tabular}{lrllllll}
\hline Genotype & No. & OS $(\% ; 95 \%$ CI $)$ & $P^{*}$ & HR $(95 \%$ CI $)$ & $P^{\#}$ & HR $(95 \%$ CI $)$ & $P^{\&}$ \\
\hline$M S H 2$ rs2303428 & & & & & & & \\
$T T$ & 234 & $68.8(62.2-74.4)$ & - & 1.00 & - & 1.00 & - \\
$T C$ & 275 & $64.3(58.2-69.8)$ & 0.126 & $1.26(0.94-1.69)$ & 0.127 & $1.19(0.88-1.59)$ & 0.256 \\
$C C$ & 67 & $50.4(37.8-61.6)$ & 0.006 & $1.70(1.14-2.54)$ & 0.009 & $1.38(0.92-2.07)$ & 0.115 \\
$T C+C C$ & 342 & $61.6(56.1-66.6)$ & 0.036 & $1.34(1.02-1.78)$ & 0.038 & $1.23(0.93-1.62)$ & 0.149 \\
$T T+C T$ (vs $C C)$ & 509 & $66.4(62.0-70.4)$ & 0.026 & $0.67(0.46-0.96)$ & 0.028 & $0.80(0.55-1.15)$ & 0.221 \\
$T R E X 1$ rs11797 & & & & & & & \\
$G G$ & 291 & $63.9(57.9-69.2)$ & - & 1.00 & - & 1.00 & - \\
$G A$ & 236 & $66.2(59.8-71.9)$ & 0.911 & $1.02(0.77-1.35)$ & 0.911 & $1.07(0.81-1.41)$ & 0.647 \\
$A A$ & 49 & $60.1(44.6-72.5)$ & 0.455 & $1.20(0.75-1.91)$ & 0.453 & $0.96(0.60-1.53)$ & 0.847 \\
$G A+A A$ & 285 & $64.9(60.6-68.9)$ & 0.737 & $1.05(0.80-1.36)$ & 0.739 & $1.04(0.80-1.36)$ & 0.749 \\
$G G+G A$ (vs $A A)$ & 527 & $65.1(59.2-70.4)$ & 0.450 & $0.84(0.54-1.32)$ & 0.454 & $1.08(0.69-1.69)$ & 0.743 \\
\hline
\end{tabular}

$O S$ overall survival, $H R$ hazard ratio, $C I$ confidence interval

*P values were calculated by the log-rank test

${ }^{\#}$ HRs in univariate analyses

${ }^{\&}$ HRs in multivariate analyses and covariates were adopted for their prognostic significance by univariate analysis, including age, histology grade and TNM stage
$C$-allele and the protein expressions of several classical biomarkers for drug-resistance. These findings indicate MSIindependent pathogenic mechanisms for MMR polymorphisms. More studies are needed to explore the function of rs2303428.

The rs2303428 $C$-allele correlates with risks of various cancers, including GC, lung cancer, endometrial cancer, and others [17, 18, 22-24]. However, the results of these studies remain conflicting rather than conclusive. A few studies also investigated the association of rs2303428 with clinical outcomes. An early study found that the rs 2303428 $C$-allele predicted tumor resectability in pancreatic cancer [26]. Zhu et al. [27] recently observed that the rs 2303428 genotype was an independent prognostic factor in hepatocellular carcinoma. Two other studies, however, failed to show an association of this polymorphism with prognosis and/or chemoradiotherapy response in rectal cancer and head and neck squamous cell carcinoma [28, 29]. Together with our results relating its positive correlation with GC outcome, these findings may indicate a tumor type-dependent effect for this polymorphism.

Heterogeneous impacts of MMR polymorphisms on the clinical outcomes of cardia and non-cardia patients were observed. Adjuvant chemotherapy even seemed to be deleterious for cardia patients with the rs2303428 TT genotype. The different molecular and clinicopathologic characteristics between cardia and non-cardia GC have been previously reported $[3,30,36]$. In addition, genome-wide association studies found different associations of genetic polymorphisms with disease risk between cardia and non-cardia GC 
Fig. 3 Overall survival in patients from the validation set by treatment status and the rs 2303428 genotypes. $F U$ fluoropyrimidines
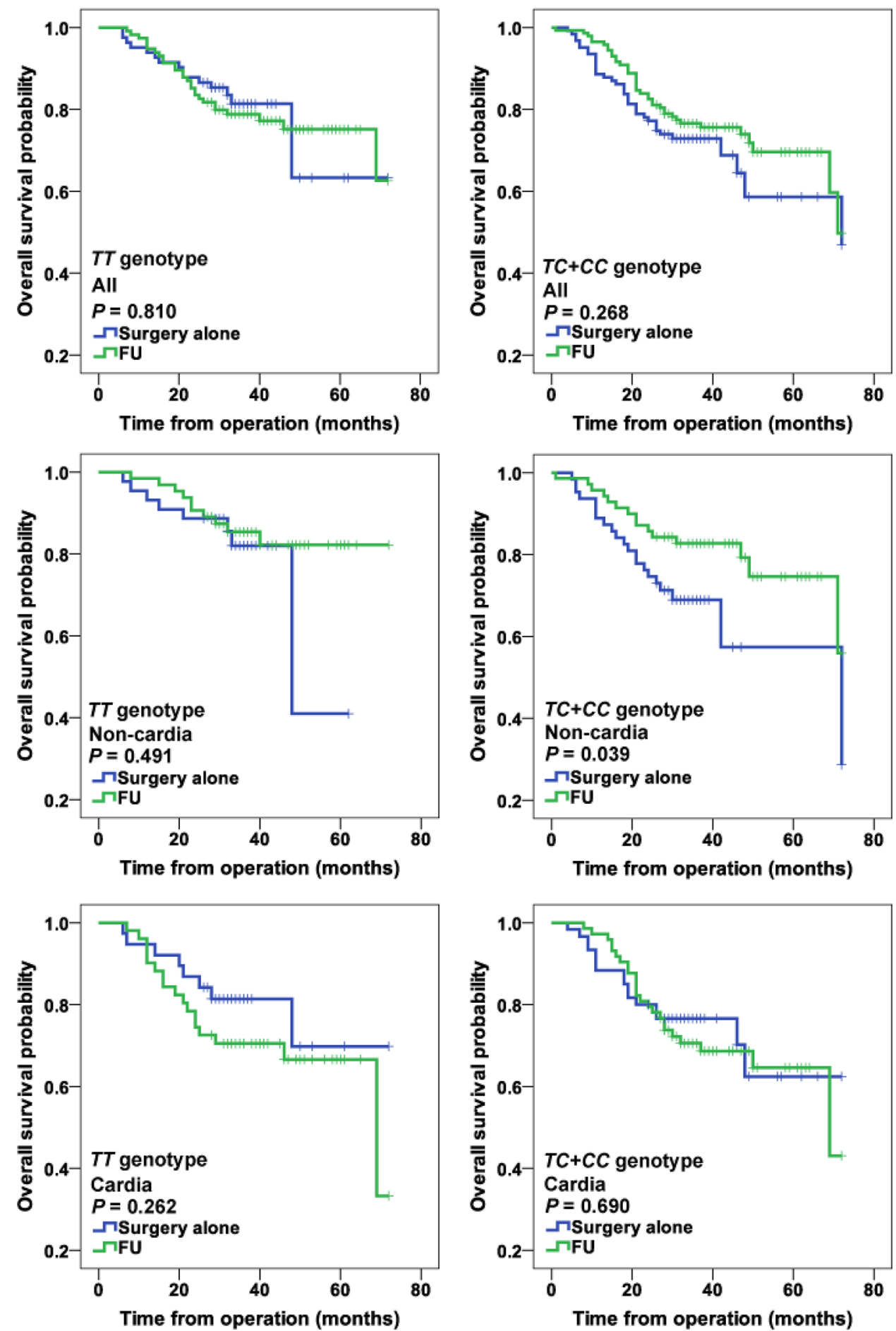

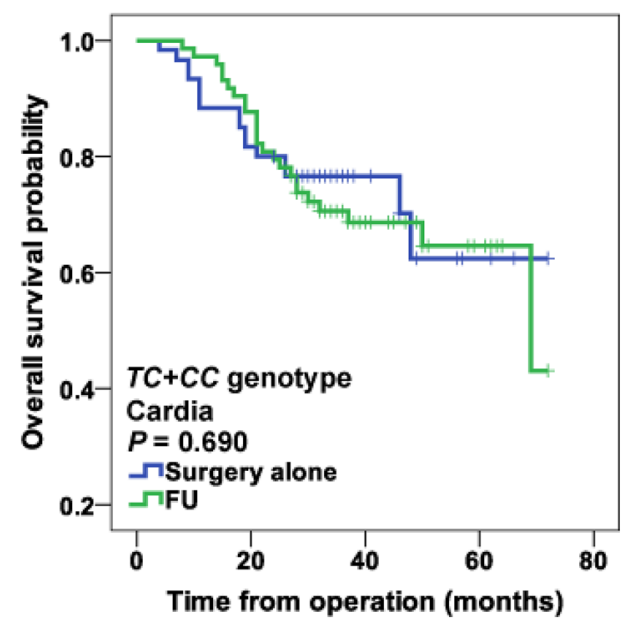

[37]. More importantly, Kim et al. [36] observed that cardia GC had poorer prognosis than non-cardia GC and tumor site was an independent prognostic predictor. Then, our findings reasonably indicate that prognostic and predictive roles of biomarkers in GC may also be dependent on tumor location.

The current study has several limitations. First, this is primarily a retrospective study based on a non-randomized selection of patients for chemotherapy versus no chemotherapy. Our results need to be confirmed by randomized prospective studies. Second, ethnic differences have been observed for some MMR genotype frequencies [20], and future studies in other countries may show some different results from ours. In addition, the intestinal and diffuse subtypes, frequently exhibiting marked differences, were unavailable in our data. However, our results according to tumor locations may indicate the different associations of 
Table 4 Associations of the rs2303428 genotypes with biomarkers of drug resistance

\begin{tabular}{|c|c|c|c|}
\hline Protein expression & $T T$ & $T C+C C$ & $P$ value \\
\hline \multicolumn{4}{|l|}{$\mathrm{TS}$} \\
\hline \multicolumn{4}{|l|}{ Non-cardia } \\
\hline Negative & $28(34.1)$ & $49(49.5)$ & \multirow[t]{2}{*}{0.038} \\
\hline Positive & $54(65.9)$ & $50(50.5)$ & \\
\hline \multicolumn{4}{|l|}{ Cardia } \\
\hline Negative & $38(50.7)$ & $50(47.6)$ & \multirow[t]{2}{*}{0.687} \\
\hline Positive & $37(49.3)$ & $55(52.4)$ & \\
\hline \multicolumn{4}{|l|}{ Total } \\
\hline Negative & $66(42.0)$ & $99(48.5)$ & \multirow[t]{2}{*}{0.220} \\
\hline Positive & $91(58.0)$ & $105(51.5)$ & \\
\hline \multicolumn{4}{|l|}{ Pgp } \\
\hline \multicolumn{4}{|l|}{ Non-cardia } \\
\hline Negative & $42(51.2)$ & $68(68.7)$ & \multirow[t]{2}{*}{0.017} \\
\hline Positive & $40(48.8)$ & $31(31.3)$ & \\
\hline \multicolumn{4}{|l|}{ Cardia } \\
\hline Negative & $43(57.3)$ & $66(62.9)$ & \multirow[t]{2}{*}{0.455} \\
\hline Positive & $32(42.7)$ & $39(37.1)$ & \\
\hline \multicolumn{4}{|l|}{ Total } \\
\hline Negative & $85(54.1)$ & $134(65.7)$ & \multirow[t]{2}{*}{0.026} \\
\hline Positive & $72(45.9)$ & $70(34.3)$ & \\
\hline \multicolumn{4}{|l|}{ ERCC1 } \\
\hline \multicolumn{4}{|l|}{ Non-cardia } \\
\hline Negative & $34(41.5)$ & $59(59.6)$ & \multirow[t]{2}{*}{0.015} \\
\hline Positive & $48(58.5)$ & $40(40.4)$ & \\
\hline \multicolumn{4}{|l|}{ Cardia } \\
\hline Negative & $31(41.3)$ & $44(41.9)$ & \multirow[t]{2}{*}{0.939} \\
\hline Positive & $44(58.7)$ & $61(58.1)$ & \\
\hline \multicolumn{4}{|l|}{ Total } \\
\hline Negative & $65(41.4)$ & $103(50.5)$ & \multirow[t]{2}{*}{0.086} \\
\hline Positive & $92(58.6)$ & $101(49.5)$ & \\
\hline \multicolumn{4}{|l|}{ HER2 } \\
\hline \multicolumn{4}{|l|}{ Non-cardia } \\
\hline Negative & 77 (93.9) & $95(96.0)$ & \multirow[t]{2}{*}{0.526} \\
\hline Positive & $5(6.1)$ & $4(4.0)$ & \\
\hline \multicolumn{4}{|l|}{ Cardia } \\
\hline Negative & $66(88.0)$ & $94(89.5)$ & \multirow[t]{2}{*}{0.748} \\
\hline Positive & $9(12.0)$ & $11(10.5)$ & \\
\hline \multicolumn{4}{|l|}{ Total } \\
\hline Negative & $143(91.1)$ & 189 (92.6) & 0.588 \\
\hline Positive & $14(8.9)$ & $15(7.4)$ & \\
\hline
\end{tabular}

$T S$ thymidylate synthetase, $P g p$ P-glycoprotein

the rs2303428 genotypes with clinical outcomes between the intestinal and diffuse subtypes, because cardia GC owned diffuse subtype more frequently than non-cardia GC [36]. Moreover, the mechanisms that are responsible for the associations of the rs 2303428 genotypes with clinical outcomes are unclear, which induces a lack of theoretical basis to use the rs2303428 genotypes clinically that is a common dilemma faced by biomarkers [7-10]. Furthermore, TNM stage still was the most powerful predictor for prognosis in GC as shown in our results, and biomarkers such as the rs2303428 genotypes alone were rather a beneficial supplement than a substitution for TNM stage. Finally, more polymorphisms in DNA repair genes need to be evaluated for clinical significance in GC.

In conclusion, this study shows that the rs 2303428 genotypes may be prognostic and predictive biomarkers in GC. If validated by prospective trials, MMR genotyping has the potential to be a convenient method to improve patient selection for adjuvant chemotherapy.

Funding This project was supported by grants from the National Natural Science Foundation of China (Grant numbers: 81301765 and 81672896), Key Project of Zhenjiang City for Health Science and Technology (SH2016038), and Project of Young Medical Talents in Jiangsu Province (QNRC2016829).

\section{Compliance with ethical standards}

Conflict of interest The authors declare that they have no conflict of interest.

Human rights statement All procedures followed were in accordance with the ethical standards of the responsible committee on human experimentation (institutional and national) and with the Helsinki Declaration of 1964 and later versions.

Informed consent Informed consent to be included in the study, or the equivalent, was obtained from all patients.

\section{References}

1. Torre LA, Bray F, Siegel RL, Ferlay J, Lortet-Tieulent J, Jemal A. Global cancer statistics, 2012. CA Cancer J Clin. 2015;65:87-108.

2. Chen W, Zheng R, Baade PD, Zhang S, Zeng H, Bray F, et al. Cancer statistics in China, 2015. CA Cancer J Clin. 2016;66:115-32.

3. Cancer Genome Atlas Research Network. Comprehensive molecular characterization of gastric adenocarcinoma. Nature. 2014:513:202-9.

4. Li GM. Mechanisms and functions of DNA mismatch repair. Cell Res. 2008;18:85-98.

5. Ribic CM, Sargent DJ, Moore MJ, Thibodeau SN, French AJ, Goldberg RM, et al. Tumor microsatellite-instability status as a predictor of benefit from fluorouracil-based adjuvant chemotherapy for colon cancer. N Engl J Med. 2003;349:247-57.

6. Sargent DJ, Marsoni S, Monges G, Thibodeau SN, Labianca R, Hamilton SR, et al. Defective mismatch repair as a predictive marker for lack of efficacy of fluorouracil-based adjuvant therapy in colon cancer. J Clin Oncol. 2010;28:3219-26.

7. An JY, Kim H, Cheong JH, Hyung WJ, Kim H, Noh SH. Microsatellite instability in sporadic gastric cancer: its prognostic role and guidance for 5-FU based chemotherapy after R0 resection. Int J Cancer. 2012;131:505-11.

8. Choi YY, Kim H, Shin SJ, Kim HY, Lee J, Yang HK, et al. Microsatellite instability and programmed cell death-ligand 1 expression 
in stage II/III gastric cancer: post hoc analysis of the classic randomized controlled study. Ann Surg. 2018. https://doi.org/10.1097/ sla.0000000000002803.

9. Oki E, Kakeji Y, Zhao Y, Yoshida R, Ando K, Masuda T, et al. Chemosensitivity and survival in gastric cancer patients with microsatellite instability. Ann Surg Oncol. 2009;16:2510-5.

10. Kim SY, Choi YY, An JY, Shin HB, Jo A, Choi H, et al. The benefit of microsatellite instability is attenuated by chemotherapy in stage II and stage III gastric cancer: results from a large cohort with subgroup analyses. Int J Cancer. 2015;137:819-25.

11. Cohen R, Hain E, Buhard O, Guilloux A, Bardier A, Kaci R, et al. Association of primary resistance to immune checkpoint inhibitors in metastatic colorectal cancer with misdiagnosis of microsatellite instability or mismatch repair deficiency status. JAMA Oncol. 2018. https://doi.org/10.1001/jamaoncol.2018.4942.

12. Klarskov L, Ladelund S, Holck S, Roenlund K, Lindebjerg J, Elebro J, et al. Interobserver variability in the evaluation of mismatch repair protein immunostaining. Hum Pathol. 2010;41:1387-96.

13. Kim ST, Cristescu R, Bass AJ, Kim KM, Odegaard JI, Kim $\mathrm{K}$, et al. Comprehensive molecular characterization of clinical responses to PD-1 inhibition in metastatic gastric cancer. Nat Med. 2018;24:1449-58.

14. Polom K, Marano L, Marrelli D, De Luca R, Roviello G, Savelli $\mathrm{V}$, et al. Meta-analysis of microsatellite instability in relation to clinicopathological characteristics and overall survival in gastric cancer. Br J Surg. 2018;105:159-67.

15. Hansen MF, Johansen J, Bjørnevoll I, Sylvander AE, Steinsbekk $\mathrm{KS}$, Sætrom P, et al. A novel POLE mutation associated with cancers of colon, pancreas, ovaries and small intestine. Fam Cancer. 2015; $14: 437-48$

16. Tournier I, Vezain M, Martins A, Charbonnier F, Baert-Desurmont $\mathrm{S}$, Olschwang $\mathrm{S}$, et al. A large fraction of unclassified variants of the mismatch repair genes MLH1 and MSH2 is associated with splicing defects. Hum Mutat. 2008;29:1412-24.

17. Wang D, Zhou J, Wang T, Li X, Li S, Chen S, et al. Polymorphisms in MSH2 gene and risk of gastric cancer, and interactions with lifestyle factors in a Chinese population. Cancer Epidemiol. 2012;36:e171-6.

18. Zhu H, Li X, Zhang X, Chen D, Li D, Ren J, et al. Polymorphisms in mismatch repair genes are associated with risk and microsatellite instability of gastric cancer, and interact with life exposures. Gene. 2016;579:52-7.

19. Raptis S, Mrkonjic M, Green RC, Pethe VV, Monga N, Chan $\mathrm{YM}$, et al. MLH1-93G $>$ A promoter polymorphism and the risk of microsatellite-unstable colorectal cancer. J Natl Cancer Inst. 2007;99:463-74

20. Xu JL, Yin ZQ, Huang MD, Wang XF, Gao W, Liu LX, et al. MLH1 polymorphisms and cancer risk: a meta-analysis based on 33 case-control studies. Asian Pac J Cancer Prev. 2012;13:901-7.

21. Xia L, Shen W, Ritacca F, Mitri A, Madlensky L, Berk T, et al. A truncated hMSH2 transcript occurs as a common variant in the population: implications for genetic diagnosis. Cancer Res. 1996;56:2289-92.

22. Jung CY, Choi JE, Park JM, Chae MH, Kang HG, Kim KM, et al. Polymorphisms in the hMSH2 gene and the risk of primary lung cancer. Cancer Epidemiol Biomark Prev. 2006;15:762-8.

23. Beiner ME, Rosen B, Fyles A, Harley I, Pal T, Siminovitch K, et al. Endometrial cancer risk is associated with variants of the mismatch repair genes MLH1 and MSH2. Cancer Epidemiol Biomark Prev. 2006;15:1636-40.

24. Wu S, Chen J, Ji Y, Liu Y, Gao L, Chen G, et al. Association between the hMSH2 IVS12-6 T $>\mathrm{C}$ polymorphism and cancer risk: A meta-analysis. Exp Ther Med. 2011;2:1193-8.
25. Kim JG, Chae YS, Sohn SK, Moon JH, Kang BW, Park JY, et al. IVS10 $+12 \mathrm{~A}>\mathrm{G}$ polymorphism in $\mathrm{hMSH} 2$ gene associated with prognosis for patients with colorectal cancer. Ann Oncol. 2010;21:525-9.

26. Dong X, Jiao L, Li Y, Evans DB, Wang H, Hess KR, et al. Significant associations of mismatch repair gene polymorphisms with clinical outcome of pancreatic cancer. J Clin Oncol. 2009;27:1592-9.

27. Zhu X, Wang Z, Qiu X, Wang W, Bei C, Tan C, et al. Rs 2303428 of MSH2 is associated with hepatocellular carcinoma prognosis in a Chinese population. DNA Cell Biol. 2018;37:634-41.

28. Yang J, Wang X, Zou SM, Li HM, Xiao Q, Feng YR, et al. Genetic variations in MLH3 and MSH2 genes are associated with the sensitivity and prognosis in locally advanced rectal cancer patients receiving preoperative chemoradiotherapy. Zhonghua Zhong Liu Za Zhi. 2018;40:433-40.

29. Nogueira GAS, Costa EFD, Lopes-Aguiar L, Lima TRP, Visacri MB, Pincinato EC, et al. Polymorphisms in DNA mismatch repair pathway genes predict toxicity and response to cisplatin chemoradiation in head and neck squamous cell carcinoma patients. Oncotarget. 2018;9:29538-47.

30. Cristescu R, Lee J, Nebozhyn M, Kim KM, Ting JC, Wong SS, et al. Molecular analysis of gastric cancer identifies subtypes associated with distinct clinical outcomes. Nat Med. 2015;21:449-56.

31. Li JC, Yang XR, Sun HX, Xu Y, Zhou J, Qiu SJ, et al. Up-regulation of Krüppel-like factor 8 promotes tumor invasion and indicates poor prognosis for hepatocellular carcinoma. Gastroenterology. 2010;139(2146-2157):e12.

32. Bartley AN, Washington MK, Colasacco C, Ventura CB, Ismaila $\mathrm{N}$, Benson AB III, et al. HER2 testing and clinical decision making in gastroesophageal adenocarcinoma: guideline from the College of American Pathologists, American Society for Clinical Pathology, and the American Society of Clinical Oncology. J Clin Oncol. 2017;35:446-64.

33. Park SH, Sohn TS, Lee J, Lim DH, Hong ME, Kim KM, et al. Phase III trial to compare adjuvant chemotherapy with capecitabine and cisplatin versus concurrent chemoradiotherapy in gastric cancer: final report of the adjuvant chemoradiotherapy in stomach tumors trial, including survival and subset analyses. J Clin Oncol. 2015;33:3130-6.

34. Noh SH, Park SR, Yang HK, Chung HC, Chung IJ, Kim SW, et al. Adjuvant capecitabine plus oxaliplatin for gastric cancer after D2 gastrectomy (CLASSIC): 5-year follow-up of an open-label, randomised phase 3 trial. Lancet Oncol. 2014;15:1389-96.

35. Liu NQ, Ter Huurne M, Nguyen LN, Peng T, Wang SY, Studd JB, et al. The non-coding variant rs1800734 enhances DCLK3 expression through long-range interaction and promotes colorectal cancer progression. Nat Commun. 2017;8:14418.

36. Kim MA, Lee HS, Yang HK, Kim WH. Clinicopathologic and protein expression differences between cardia carcinoma and noncardia carcinoma of the stomach. Cancer. 2005;103:1439-46.

37. Hu N, Wang Z, Song X, Wei L, Kim BS, Freedman ND, et al. Genome-wide association study of gastric adenocarcinoma in Asia: a comparison of associations between cardia and non-cardia tumours. Gut. 2016;65:1611-8.

Publisher's Note Springer Nature remains neutral with regard to jurisdictional claims in published maps and institutional affiliations. 